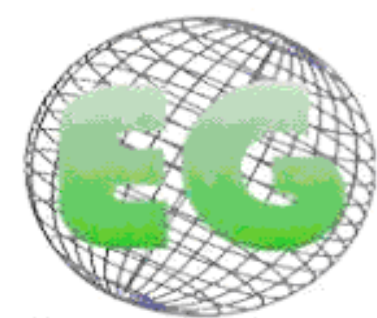

ISSN 1695-6141 N'22
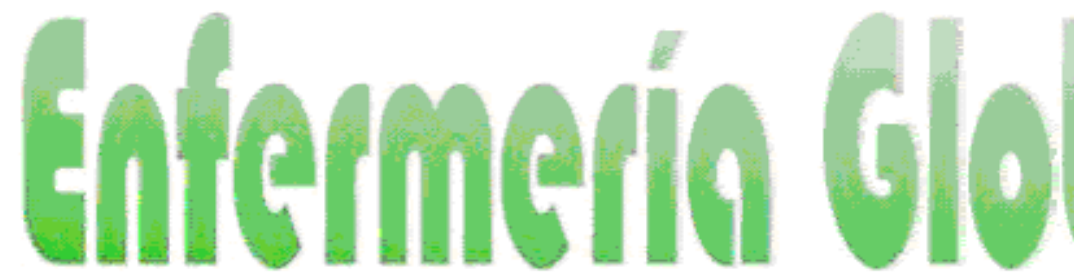

Revista electrónica trimestral de Enfermería

www.um.eslegloball

Abril 2011

\title{
PRINCIPALES CAUSAS ASOCIADAS AL TRAUMATISMO CRANEOENCEFÁLICO EN ANCIANOS
}

PRINCIPAIS CAUSAS ASSOCIADAS AO TRAUMATISMO CRANIOENCEFÁLICO EM IDOSOS

\section{"Pinheiro, Al., *De Almeida, FM., **Barbosa, IV., ${ }^{* * *}$ Mesquita Melo,E., "***Borges Studart, RM., ****De Figueiredo Carvalho, ZM.}

\author{
*Enfermeira. **Aluna do Doutorado em Enfermagem da Universidade Federal do Ceará (UFC). Professora da \\ Universidade de Fortaleza(UNIFOR). ${ }^{* * *}$ Doutora em Enfermagem. Professora da UNIFOR. ${ }^{* * *}$ Mestre em

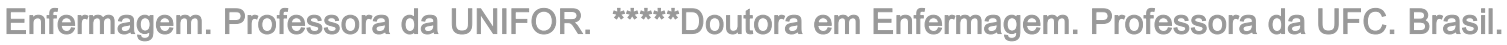

(Trabalho de conclusão do curso de Graduação da Universidade de Fortaleza.)

\author{
Palabras clave: Enfermería; Trauma cranoencefálico; Anciano \\ Palavras-chave: Enfermagem; Trauma Cranioencefálico; Idoso. \\ Keywords: Nursing; Traumatic brain injury; Elderly
}

\section{RESUMEN}

Estudios demuestran un aumento en el número de traumas en ancianos, lo que puede estar asociado al crecimiento de esta población. El objetivo del estudio fue evaluar las características de los ancianos afectados por trauma craneoencefálico (TCE) e identificar las causas asociadas a dicho traumatismo en ancianos. Estudio exploratorio descriptivo, realizado con 41 ancianos, víctimas de TCE, atendidos en hospital público, referencia en trauma, localizado en Fortaleza-Ceará. Los datos fueron colectados en los meses de febrero y marzo de 2009, por medio de un guión de entrevista estructurado, conteniendo datos de identificación y cuestiones relacionadas a las características del accidente que ocasionó el TCE. Los aspectos éticos y legales fueron contemplados. La mayoría de los participantes es de sexo masculino (85,3\%), en situación económicamente activa; $75,7 \%$ residen con familiares. La principal causa del TCE son las caídas (34,2\%). Respecto al lugar donde ocurrió el accidente que generó el TCE, $23(56,1 \%)$ fue en la calle. Los traumatismos craneoencefálicos en los ancianos constituyen un gran problema de salud pública, siendo fundamental la prevención de accidentes en dicha población.

\section{RESUMO}

Estudos demonstram um aumento no número de traumas em idosos, o que pode estar associado ao crescimento desta população. O objetivo do estudo foi avaliar as características dos idosos afetados por trauma cranioencefálico (TCE) e identificar as causas associadas ao referido traumatismo em idosos. Estudo exploratório descritivo, realizado com 41 idosos, vítimas de TCE, atendidos em 
hospital público, referência em trauma, localizado em Fortaleza-Ceará. Os dados foram coletados nos meses de fevereiro e março de 2009, por meio de um roteiro de entrevista estruturado, contendo dados de identificação e questões relacionadas às características do acidente que ocasiono o TCE. Os aspectos éticos e legais foram contemplados. A maioria dos participantes é de sexo masculino (85,3\%), em situação economicamente ativa; $75,7 \%$ moram com familiares. A principal causa do TCE são as quedas (34,2\%). Respeito ao local onde aconteceu o acidente que gerou o TCE, 23 (56,1\%) foi na rua. Os traumatismos cranioencefálico nos idosos constituem um grande problema de saúde pública, sendo fundamental a prevenção de acidentes nesta população.

\section{ABSTRACT}

Research shows an increase the trauma occurrences in the elderly, associated to the ñarger elderly population. This study aimed to evaluate characteristics of the elderly and to identify the causes associated with traumatic brain injury (TBI) in the elderly. This is an exploratory descriptive study, carried out with 41 elderly TBI victims, treated for trauma in a public hospital in Fortaleza-Ceará. Data were collected in February and March 2009, through a structured interview containing identification data and questions related to the characteristics of the accident that caused TBI. The ethical and legal aspects were guaranteed. Most of the participants were male (85.3\%), economically active; $75.7 \%$ live with relatives. The main cause of TBI was a fall (34.2\%). Concerning the place where the accident that generated TBI occurred, 23 (56.1\%) were in the street. Traumatic brain injury in elderly is a big problem in public health, so its prevention is highly important.

\section{INTRODUCCIÓN}

Con el avance de la ciencia y el crecimiento cada vez mayor de las tecnologías dirigidas a la salud de las personas, en los últimos años se observa un aumento de la población anciana, haciéndose necesario, también, una mayor capacitación de los profesionales dedicados a la atención de esta población.

El envejecimiento es una fase de la vida que conlleva varios cambios y muchos ancianos no están preparados para enfrentar esta realidad. Para que se pueda envejecer con salud es necesario que el individuo sea capaz de adaptarse a determinadas situaciones como: pérdidas físicas, sociales y emocionales.

El envejecimiento es definido como el fenómeno en el proceso de vida que, como en la infancia, la adolescencia y la madurez, pasa por cambios biopsicosociales específicos, asociados al paso del tiempo ${ }^{(1)}$.

El proceso de envejecer provoca cambios en el organismo como la disminución de la agudeza visual y auditiva, de la estatura, de la masa muscular, de la cantidad de agua corporal y en la velocidad de los reflejos; alteración en el peso corporal; aumento de la grasa; retraso y/o dificultad en el aprendizaje y en la memoria y el uso de medicamentos. Otros sistemas orgánicos también ven disminuidas de sus funciones: renal, pulmonar, cardiovascular, osteomuscular y endocrino. La movilidad queda afectada llevando a una marcha lentificada que es la mayor responsable por caídas en los ancianos ${ }^{(2)}$.

La longevidad forma parte del sueño de la población mundial lo que hace que las personas modifiquen su estilo de vida incluyendo prácticas saludables como actividad física, alimentación equilibrada, menos estrés en el trabajo, mejorar el sueño buscando la satisfacción en su día a día. La mejoría de las condiciones de saneamiento e infraestructura básica, el progreso de la medicina, de la cirugía, de la tecnología y salud pública, permitió 
avances en los recursos y en las técnicas diagnosticadas, asistenciales y terapéuticas, elevando la expectativa de vida de forma extraordinaria.

Debido al crecimiento de la población anciana, hubo un aumento en el número de traumas en esta población. Este aumento ocurrió debido al estilo de vida más activo del anciano exponiéndolo al riesgo de accidentes.

Aproximadamente $75 \%$ de las muertes como consecuencia de caídas en los Estados Unidos ocurrió en 14\% de la población de más de 65 años de edad, y el índice de mortalidad aumenta dramáticamente después de los 70 años, principalmente en hombres ${ }^{(3)}$.

Los accidentes y la violencia configuran un problema de salud pública de gran magnitud y trascendencia, con fuerte impacto en la morbilidad y en la mortalidad de la población. En el conjunto de las lesiones como consecuencia de causas externas, el trauma craneoencefálico (TCE) se destaca en términos de magnitud tanto entre muertes como en heridos, siendo una de las lesiones más frecuentes.

Las víctimas que sobreviven al TCE pueden presentar deficiencias e incapacidades que pueden ser temporales o permanentes, interfiriendo en la capacidad del individuo para desempeñar sus funciones ${ }^{(4)}$.

En 2004, en Brasil, 16.789 ancianos murieron víctimas de accidentes principalmente de tránsito y violencia. Lo que significa que cerca de 46 ancianos mueren diariamente siendo $65 \%$ del sexo masculino. Mientras que en 1991 el número de muertes de ancianos por violencia o accidentes era de 104 personas por grupo de 100 mil, en 2004 este número subió para 110 por 100 mil. Este aumento se debe a accidentes de tránsito, caídas, suicidios y violencia doméstica ${ }^{(5)}$. El creciente aumento de la violencia y de las caídas generó una demanda significativa de ancianos atendidos en los hospitales de referencia en trauma.

En las últimas décadas se observa un crecimiento expresivo en la población anciana en el mundo y más particularmente en los países en desarrollo ${ }^{(6)}$. Por ello, este estudio es relevante pues permitirá conocer las causas del trauma craneoencefálico en ancianos, con el fin de proporcionar una mejor asistencia y un mayor control de sus complicaciones y una posible prevención de las causas en el ámbito de actuación de la salud pública.

El estudio tuvo como objetivo evaluar las características de los ancianos afectados por trauma craneoencefálico e identificar las principales causas de traumatismo craneoencefálico en la población anciana.

\section{MATERIAL Y MÉTODOS}

Se trata de un estudio exploratorio y descriptivo con abordaje cuantitativo. Los estudios descriptivos permiten explorar una situación no conocida, de la cual se necesitan mayores informaciones sobre cierto asunto, exploran la realidad e identifican las características y regularidades de los fenómenos ${ }^{(7)}$.

Fue realizado en un Hospital Público de referencia en atención a las víctimas de trauma en la ciudad de Fortaleza-Ceará. La muestra estuvo compuesta por 41 ancianos atendidos con TCE durante el periodo de enero a marzo de 2009. Los criterios de inclusión fueron: estar internado más de 48 horas con diagnóstico médico definido, tener edad igual o superior a 60 años, estar en condiciones neurológicas para responder al cuestionario y tener cuidador de 
la familia o contratado que acepte participar del estudio mediante la firma de la declaración de consentimiento.

Los datos fueron colectados en los meses de febrero y marzo de 2009, por medio de un guión de entrevista conteniendo datos de identificación y cuestionamientos relacionados con las características del accidente que ocasionó el TCE. Para el análisis, los datos de los pacientes fueron transcritos y tabulados en una planilla del programa Excel del Windows $X P$ Profesional siendo organizados en tablas y gráficos que fueron interpretados $y$ fundamentados conforme a las publicaciones sobre el tema.

Los aspectos éticos y legales fueron contemplados. El proyecto fue encaminado al Comité de Ética e Investigación de la Institución. La participación en el estudio fue voluntaria, con libertad de de asistencia en cualquier momento. Los participantes firmaron una declaración de consentimiento, demostrando interés en participar del estudio ${ }^{(8)}$.

\section{RESULTADOS}

Observamos que gran parte de los participantes 33 (80,5\%) fue de sexo masculino, y la mayoría se encontraba en la franja de edad de 60 a 69 años (41,5\%); ocho ancianos $(19,5 \%)$ eran de sexo femenino, siendo tres $(7,3 \%)$ en la franja de edad de 60 a 69 años y tres $(7,3 \%)$ en la franja de 80 años o más.

FIGURA 1: Distribución de los pacientes según sexo y franja de edad. Fortaleza, 2009.

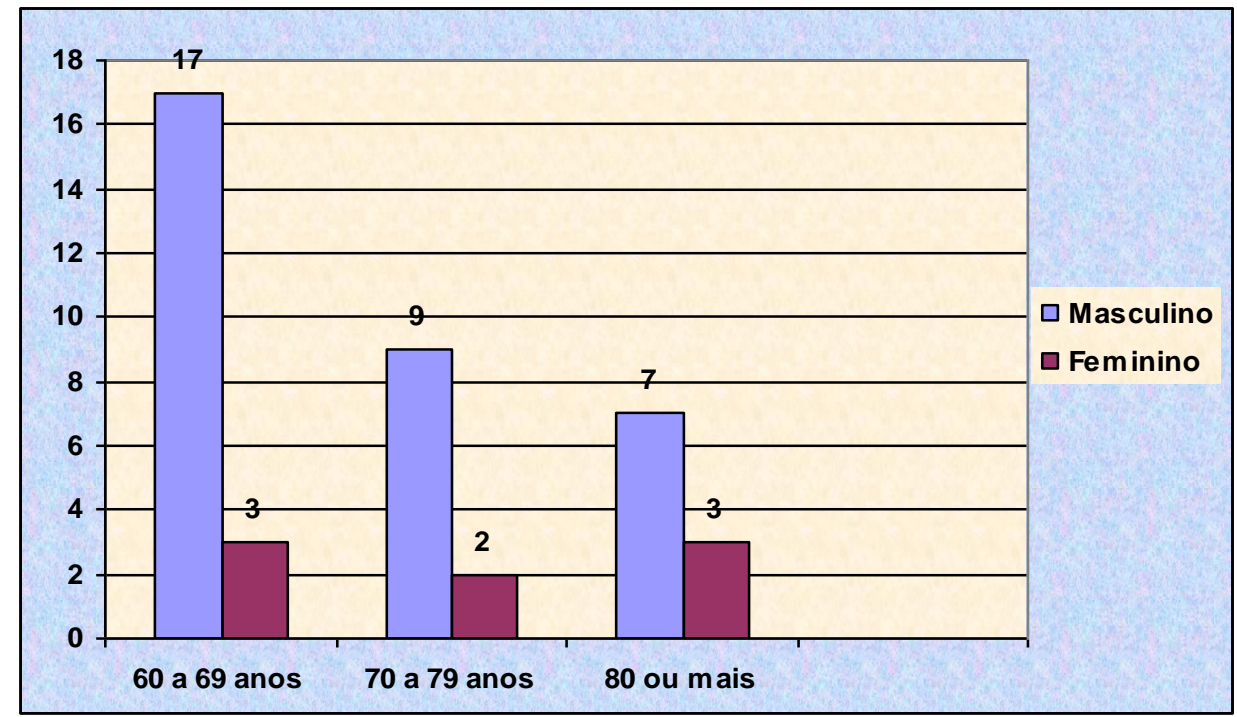

En cuanto al nivel de escolaridad, $49 \%$ de los ancianos se denominaron analfabetos $34 \%$ no consiguieron completar el $1^{\circ}$ grado, $15 \%$ completaron el $1^{\circ}$ grado y $2 \%$ completó el $2^{\circ}$ grado. 
FIGURA 2: Distribución de los pacientes según grado de instrucción.

Fortaleza, 2009.

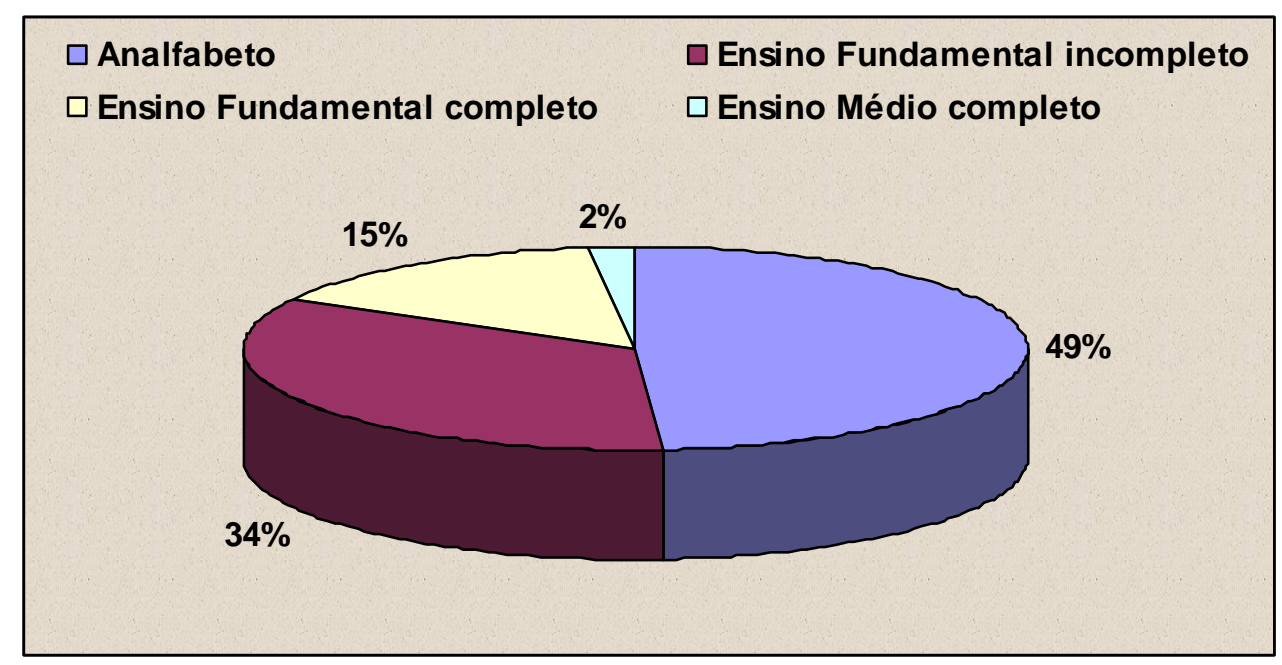

En relación al salario mensual recibido por ese anciano fue encontrado que el $80 \%$ de la muestra recibe 1 salario mínimo, $15 \%$ recibe de 2 a 4 salarios y solamente $5 \%$ recibe más de 4 salarios por mes

FIGURA 3: Distribución de los pacientes según renta mensual. Fortaleza, 2009.

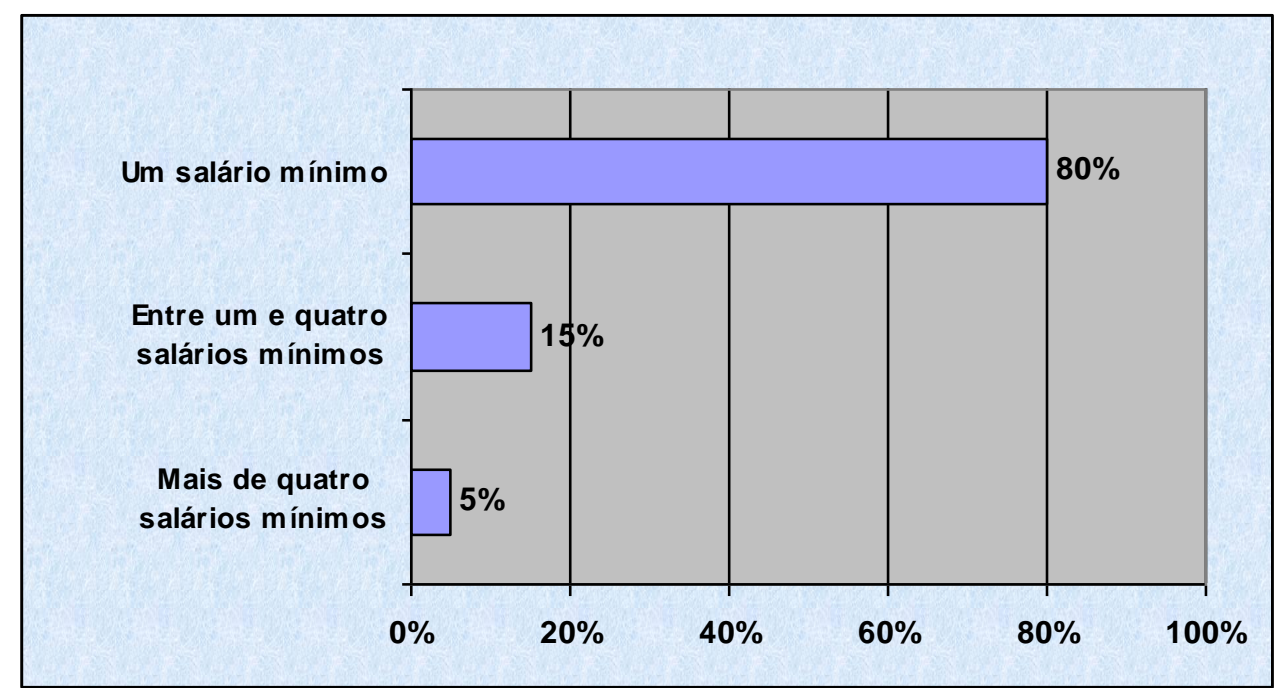

Evidenciamos en el estudio que 36,59\% (15) de los ancianos residían con la esposa, 31,7\% (13) con hijos, $24,39 \%$ (10) viven solos y $7,32 \%$ (3) viven con otros: que incluyen amigos, nietos y hermana 
FIGURA 4: Distribución de los pacientes según los familiares con los que conviven. Fortaleza, 2009.

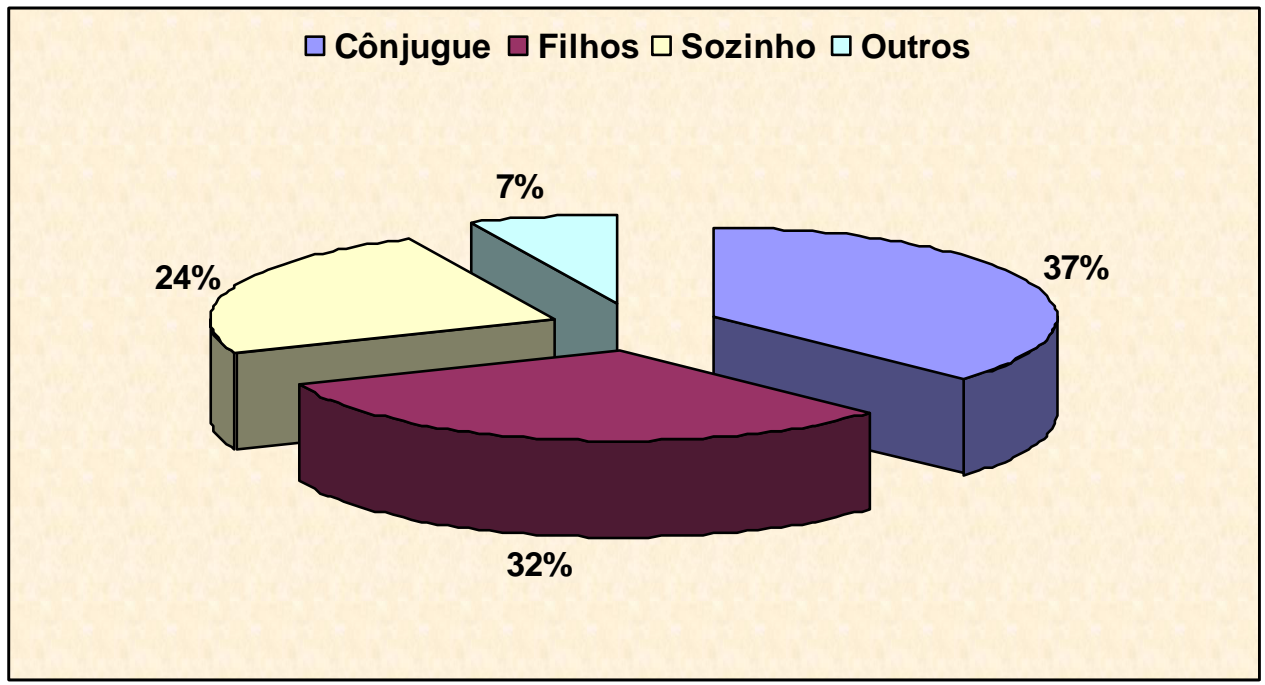

En cuanto a la procedencia constatamos que 19 ancianos, lo que corresponde al $46 \%$ eran de la capital del estado, 8 ancianos, correspondiente al 20\%, provenían del área metropolitana y 14 , lo que es equivalente al $34 \%$, se desplazaron del interior para la capital en busca de atención.

FIGURA 5: Distribución de los pacientes según procedencia.

Fortaleza, 2009.

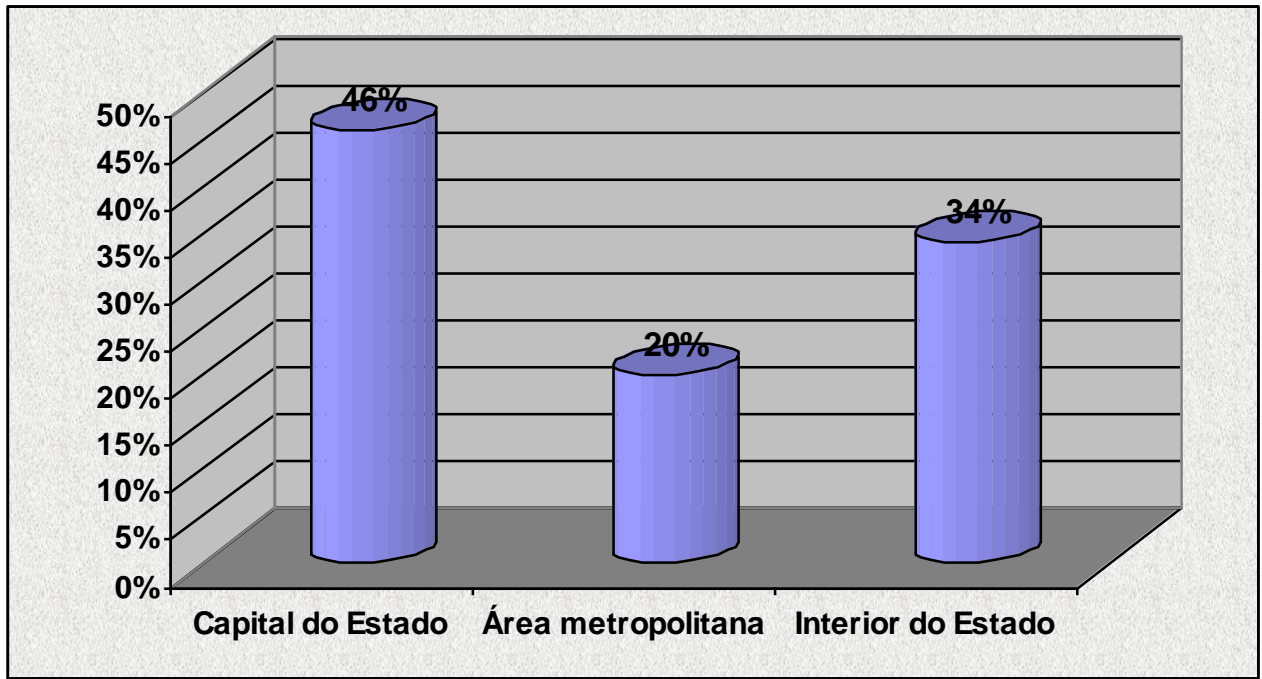

La mayoría de los pacientes se accidentaron por caída $(34,2 \%)$, seguido por atropello $(19,5 \%)$, caída de la propia altura $6(14,6 \%)$, accidente de moto $(12,2 \%)$, accidente de bicicleta $3(7,3 \%)$, agresión $3(7,3 \%)$ y accidente automovilístico 2 (4,9\%), según muestra la Tabla 1. 
TABELA 1 - Distribución de los pacientes en cuanto al tipo de accidente. Fortaleza, 2009.

\begin{tabular}{lcc}
\hline Tipo de acidente & $N$ & $\%$ \\
\hline Quedas & 14 & 34,2 \\
Atropelamento & 08 & 19,5 \\
Queda da própria altura & 06 & 14,6 \\
Moto & 05 & 12,2 \\
Agressão & 03 & 7,3 \\
Bicicleta & 03 & 7,3 \\
Automobilístico & 02 & 4,9 \\
\hline Total & 41 & 100 \\
\hline
\end{tabular}

Respecto al lugar donde ocurrió el accidente que generó el TCE, 23 (56,1\%) fue en la calle, siendo prestada atención prehospitalaria a 15 de estos pacientes y 18 (43,9\%) ocurrieron en el domicilio, con una atención prehospitalaria prestada a siete $(17 \%)$ de los pacientes.

FIGURA 6: Distribución de los pacientes según lugar del accidente y atención pre-hospitalaria. Fortaleza, 2009.

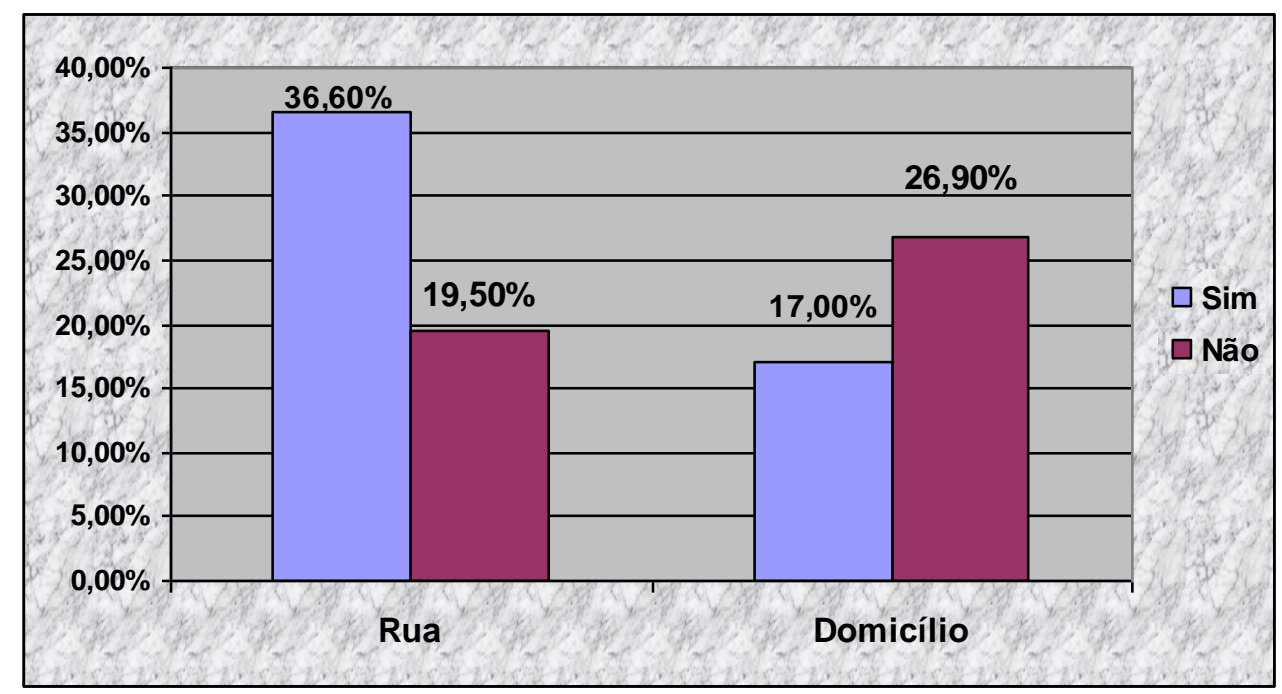

A pesar de que la mayoría de los pacientes recibió atención prehospitalaria en el propio lugar del accidente, un porcentaje relevante de pacientes no fueron atendidos, lo que pudo contribuir a la gravedad del cuadro y surgimiento de complicaciones y secuelas.

\section{DISCUSIÓN}

Los traumatismos craneoencefálicos en los ancianos constituyen un gran problema de salud pública, siendo muy frecuentes debido a algunas debilidades comunes en esta fase de la vida, como: reflejos disminuidos, memoria alterada, enfermedades sobrevenidas, marcha lenta y comprometimiento sensorial con deficiencias de visión y audición, resultando en tiempo de reacción alterado.

En lo que se refiere a la franja de edad y sexo, la más afectada entre los ancianos estudiados fue la que se encuentra entre 60 y 69 años de sexo masculino, corroborando la 
literatura ${ }^{(9,10)}$. Podemos inferir que los hombres se exponen más a riesgos, tal vez por el contexto socio cultural en que están insertos.

En relación a la escolaridad, la mayoría no poseía enseñanza fundamental completa, habiendo un número significativo de analfabetos. Según Marin et al, el analfabetismo en el anciano representa una realidad de los países en desarrollo como, por ejemplo, Brasil ${ }^{(11)}$.

La baja escolaridad lleva al anciano a someterse a subempleos que no proporcionan una estabilidad financiera, haciendo que muchos busquen actividades paralelas para complementar la renta familiar. Actividades que colocan en riesgo el bienestar físico, con alta probabilidad de caídas y accidentes.

En relación a los familiares con los que conviven, observamos que la mayoría de los pacientes reside con el cónyuge y/o con los hijos, lo que puede reducir el riesgo de accidentes en el domicilio, ya que la familia, algunas veces, está presente supervisando sus actividades diarias. Cuando viven solos se adaptan a las actividades, pero son más vulnerables a las caídas.

En cuanto a la procedencia de los pacientes, los datos demuestran que un porcentaje significativo (46\%) de los entrevistados proviene de la capital del Estado. Los pacientes se desplazaron del interior del Estado en busca de atención debido a la falta de estructura de ayuda del hospital de base, provocando una ocupación notable en el hospital de referencia estadual para víctimas de traumas.

El estudio reveló que la mayor causa de TCE en los ancianos fue la caída y en segundo lugar el atropellamiento. Souza e Iglesias y Siqueira et al en sus estudios afirmaron que la caída es el mecanismo de lesión más frecuente entre los ancianos y que sus consecuencias representan un gran problema de salud ${ }^{(9,12,13)}$. La disminución de la visión contribuye a caídas recurrentes y cuanto mayor es la pérdida visual, mayor el riesgo de caídas ${ }^{(11)}$.

La falta de estructura principalmente en los domicilios, que no siempre están adaptados para recibir a los ancianos, muchas veces contribuye a que los ancianos sufran caídas. Deben hacerse cambios para promover el andar seguro para el anciano, cambios como retirada de alfombras, colocación de barras de seguridad, evitar suelos resbaladizos, mantener una buena luminosidad en el interior de la residencia favoreciendo la integridad del anciano en el ambiente doméstico.

Otro factor que influye en las caídas y también en el atropello, que fue la segunda causa de TCE en los ancianos, es la precariedad de las vías públicas que todavía es un factor predominante cuando se habla de ocurrencias con ancianos, este al salir por las calles necesita acompañamiento y muchas veces la familia no hace esta supervisión, acabando este muy expuesto a peligros totalmente previsibles.

En el tránsito, los ancianos en Brasil pasan por una combinación de desventajas: dificultades de movimientos propias de la edad, se suman a mucha falta de respeto e incluso a violencias infringidas por conductores y a negligencias del poder público. La falta de medidas preventivas de tránsito como el respeto al paso de peatones así como de otras señalizaciones, pueden ser consideradas como responsables por el gran número de accidentes con ancianos.

Con la mejoría de las condiciones de salud y soporte preventivo para esta franja de edad, los ancianos presentan una vida más saludable y activa, determinando mayor exposición a 
accidentes externos, como atropellos y accidentes automovilísticos, lo que asociado a las características fisiológicas propias de esta franja de edad muestra un comportamiento diferente de los demás grupos ${ }^{(14)}$.

En relación a la atención prehospitalaria, en el estudio encontramos que la prevalencia fue de pacientes que tuvieron acceso a dicha atención y en su mayor parte fueron atendidos en la calle. Cabe resaltar que después del surgimiento de la atención prehospitalaria, la expectativa de vida de los pacientes aumentó y también se redujo el agravamiento de las lesiones y la ocurrencia de lesiones secundarias gracias al equipo de primeros socorros especializados.

La atención adecuada y el tiempo transcurrido entre el accidente y la admisión hospitalaria son factores relevantes para reducir la mortalidad de las víctimas de lesiones por accidentes y violencias. La primera hora después de la ocurrencia es considerada el tiempo crítico para la institución del tratamiento que modificará el pronóstico ${ }^{(15)}$.

El avance tecnológico proporciona mejoras en la calidad de vida en general y en los cuidados a la salud en particular, siendo esta la causa más citada para el envejecimiento poblacional en todo el mundo. El progreso innegable de la medicina ha permitido que la población anciana alcance patrones de bienestar ${ }^{(16)}$.

La enfermera tiene como función proporcionar al anciano medios que permitan la readquisición de la auto-estima y la auto-confianza para el restablecimiento de su independencia, proporcionando así un rápido retorno a las actividades de la vida diaria y una mejor calidad de vida. De acuerdo con Campos et al, la enfermería geriátrica debe dar apoyo educativo a los ancianos, víctimas de caídas, animándolos para el desarrollo de patrones de autocuidado y promoción de la independencia ${ }^{(17)}$.

\section{CONCLUSIONES}

Los traumatismos craneoencefálicos en los ancianos constituyen un gran problema de salud pública, siendo muy frecuentes debido a algunas debilidades comunes en esta fase de la vida. En este sentido, es fundamental la prevención de accidentes en la población anciana. La mayoría de los participantes era de sexo masculino, se encontraba en la franja de edad de 60 a 69 años, no poseía enseñanza fundamental completa, era procedente de la capital del estado, tenía una renta mensual de hasta un salario mínimo y residía con el cónyuge y/o con hijos.

Identificamos que la principal causa del TCE son las caídas, seguido por atropellos. El lugar predominante de los accidentes fue la calle, siendo que la mayoría de los pacientes recibieron atención prehospitalaria en el lugar del accidente.

Forma parte de la rutina del enfermero realizar examen físico y evaluación neurológica, ya que dentro del equipo es el que está más presente en el día a día del paciente, pudiendo observar cambios de comportamiento y motores y siendo capaz de intervenir precozmente. El equipo deberá estar apto para interpretar las correctas alteraciones ocurridas en el anciano después del trauma, tomando en consideración que el anciano presenta alteraciones específicas de la edad.

Es de fundamental importancia que los gobernantes actúen junto con la comunidad proporcionando una mejoría de las condiciones de saneamiento e infraestructura básicas; 
faciliten el acceso a consultas y exámenes que posibiliten diagnósticos precoces permitiendo un rápido y efectivo tratamiento.

Enfatizamos que las medidas preventivas deben ser abordadas dentro de la salud pública y en el contexto hospitalario. El enfermero es un educador por excelencia, teniendo como meta promover la salud y orientar a sus pacientes para prácticas que lleven a una mejor calidad de vida, actuando también junto con la familia de este anciano favoreciendo un ambiente más tranquilo y seguro.

Desde esta perspectiva, esperamos que los resultados de esta investigación puedan contribuir con los profesionales de la salud que actúan en el cuidado directo a los ancianos que sufren TCE, en las áreas de gerontología, emergencias de los hospitales y en la prevención de posibles accidentes en las casas de apoyo a ancianos, así como revelar para la institución del estudio la casuística de TCE que afecta a la población anciana atendida.

\section{REFERENCIAS}

1 Vieira EB. Manual de Gerontologia: um guia teórico prático para profissionais, cuidadores e familiares. $2^{\text {a }}$ ed. Rio de Janeiro: Revinter, 2004.

2 Duncan BB, Schmidt MI, Giugliani ERJ. e [et al.]. Medicina ambulatorial: condutas de atenção primária baseada em evidências. 3ªed. Porto Alegre: Artmed, 2004.

3 Frame SB. Atendimento pré-hospitalar ao traumatizado: básico e avançado. Rio de Janeiro: Elsevier, 2004.

4 Brasil. Biblioteca Virtual em Saúde. Idosos no mundo. Brasília: Ministério da Saúde, 2005. Disponível em: http://bvsms.saude.gov.br/bvs/exposicoes/idoso/idosom.html. Acesso em 12/09/08.

5 Minayo MCS. Violência contra idosos: relevância para um velho problema. Caderno Saúde Pública. 19 (3): 783-791, Jun, 2003.

6 Soares JL, Faro ACM. Trauma ortopédico em idosos: uma revisão de literatura. 2 (7): 2005. Disponível em: http://revistas.um.es/eglobal/article/viewFile/491/482. Acesso em 20/07/09.

7 Minayo MCS. O desafio do conhecimento: pesquisa qualitativa em saúde. 8a ed. São Paulo: HUCITEC; Rio de Janeiro: ABRASCO, 2004.

8 Brasil. Conselho Nacional de Saúde. Resolução n196/96. Decreto n 93. 933 de janeiro de 1987. Estabelece critérios sobre pesquisa envolvendo seres humanos. Brasília: 1996.

9 Souza J, Iglesias ACRG. Trauma no idoso. Rev Assoc Med Bras, Rio de Janeiro. 48 (1): 79-86, 2002.

10 Feitoza DS, Freitas MC, Silveira RE. Traumatismo crânioencefálico: diagnósticos de enfermagem a vítimas atendidas em UTI. Revista Eletrônica de Enfermagem, 6 (2): 223-233, 2004.

11 Marin MJS, Amaral FS, Martins IB, Bertassi VC. Identificando os fatores relacionados ao diagnóstico de enfermagem "risco de quedas" entre idosos. Rev. Bras. Enferm., 57 (5), set./out., 2004.

12 Siqueira FV, Facchini LA, Piccini RX, Tomasi E, Thumé E, Silveira D, Vieira V, Hallal PC. Prevalência de quedas em idosos e fatores associados. Rev. Saúde Pública. 41 (5), 2007.

13 Piras C, Forte LV, Peluso CM, Lima EM, Prandini MN. Estudo epidemiológico do TCE em Unidade de Terapia Intensiva Geral como resultado da adesão ao Latin American Brain Injury Consortium. Revista Brasileira de Terapia Intensiva. 15 (3), 2004.

14 Katz M, Okuma MAA, Santos ALG, Guglielmetti CLB, Sakaki MH, Zumiotti AV. Epidemiologia das lesões traumáticas de alta energia em idosos. Acta ortop. bras., 16 (5), 2008. 
15 Ladeira RM, Barreto SM. Fatores associados ao uso de serviço de atenção pré-hospitalar por vítimas de acidentes de trânsito. Cad. saúde pública, 24 (2): 287-294, 2008.

16 Fonseca MM, Gonçalves HS. Violência contra o idoso: Suportes legais para a intervenção. Interação psicol., 7 (2): 121-128, 2003.

17 Campos JFS, Poletti NAA, Rodrigues CDS, Garcia TPR, Angelini JF, Dollinger APAV, Ribeiro RCHM. Trauma em idosos atendidos no pronto atendimento da emergência do Hospital de Base. Arq Ciênc. Saúde, 14 (4): 193-197, 2007. 